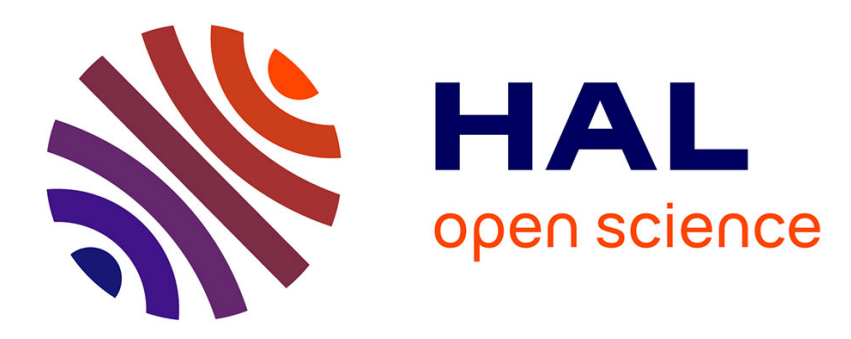

\title{
Memory effects in a random walk description of protein structure ensembles
}

Gerald R. Kneller, Konrad Hinsen

\section{To cite this version:}

Gerald R. Kneller, Konrad Hinsen. Memory effects in a random walk description of protein structure ensembles. Journal of Chemical Physics, 2019, 150 (6), pp.064911. 10.1063/1.5054887 . hal02117662

\section{HAL Id: hal-02117662 \\ https://hal.science/hal-02117662}

Submitted on 2 May 2019

HAL is a multi-disciplinary open access archive for the deposit and dissemination of scientific research documents, whether they are published or not. The documents may come from teaching and research institutions in France or abroad, or from public or private research centers.
L'archive ouverte pluridisciplinaire HAL, est destinée au dépôt et à la diffusion de documents scientifiques de niveau recherche, publiés ou non, émanant des établissements d'enseignement et de recherche français ou étrangers, des laboratoires publics ou privés. 


\title{
Memory Effects in a Random Walk Description of Protein Structure Ensembles
}

\author{
Gerald R. Kneller ${ }^{1,2,3}$ and Konrad Hinsen ${ }^{1,3}$ \\ ${ }^{1}$ Centre de Biophys. Moléculaire, CNRS; Rue Charles Sadron, 45071 Orléans, France \\ ${ }^{2}$ Université d'Orléans; Chateau de la Source-Av. du Parc Floral, 45067 Orléans, France and \\ ${ }^{3}$ Synchrotron Soleil; L'Orme de Merisiers, 91192 Gif-sur-Yvette, France
}

\begin{abstract}
In this paper we show that ensembles of well-structured and unstructured proteins can be distinguished by borrowing concepts from non-equilibrium statistical mechanics. For this purpose, we represent proteins by two different polymer models and interpret the resulting polymer configurations as random walks of a diffusing particle in space. The first model is the trace of the $C_{\alpha}$-atoms along the protein main chain and the second their projections onto the protein axis. The resulting trajectories are subsequently analyzed using the theory of the Generalized Langevin Equation. Velocities are replaced by displacements relating consecutive points on the discrete protein axes and equilibrium ensemble averages by averages over appropriate protein structure ensembles. The resulting displacement autocorrelation functions resemble those of velocity autocorrelation functions of simple liquids and display a minimum, which can be related to the lengths of secondary structure elements. This minimum is clearly more pronounced for well-structured proteins than for unstructured ones and the corresponding memory function displays a slower decay, indicating a stronger "folding memory".
\end{abstract}

PACS numbers:

Keywords:

\section{INTRODUCTION}

The protein structure-function relationship is one of the basic concepts in structural biology and it has for several decades driven the determination of protein structures by X-ray and neutron crystallography as well as by nuclear magnetic resonance (NMR) techniques. It was soon recognised that protein function requires dynamic structures, 1,4 and one can observe a change in paradigm over the last years, admitting that protein function does not necessarily require well-defined structures. One speaks here of intrinsically disordered proteins (IDP), where the term "disorder" describes the absence of well-defined secondary structure elements and may concern the whole protein or parts of it $[5]$ In contrast to well-structured proteins, for which more than 140000 structures can be found at present in the Protein Data Bank ${ }^{9}$, much less is known about the possible conformations of IDPs. Information comes here essentially from computer-generated models which are compatible with experimental data from structural NMR and small angle diffraction techniques. Corresponding data bases are being built up $\frac{10[11}{1}$ and start to become exploitable from a statistical point of view. One can therefore search for criteria that allow a distinction between structured and unstructured proteins on a purely statistical basis. Since protein structure data bases contain structures and structure ensembles of different proteins, such statistical models should be based on the conformation of the protein main chain only. The simplest example is the polymer chain model by W. Kuhn, $\frac{12]}{\text { which }}$ consists of equidistantly spaced point-like monomers and which can be transposed to proteins by associating each $C_{\alpha}$-atom along the protein main chain with a monomer of the Kuhn chain. We note here that that due to the rigid geometry of peptide bonds, the distances between consecutive $C_{\alpha}$-atoms in proteins have an almost constant value of $0.4 \mathrm{~nm}$. The polymer configurations in Kuhn's model are random chains, where all monomers are placed randomly at the fixed distance to their respective predecessor along the polymer chain. These freely jointed chains lead to a Gaussian model for the probability distribution of finding a monomer at a distance $\mathbf{r}$ from a given monomer and they can be interpreted as trajectories of Brownian particles whose subsequent positions in time correspond to the monomer positions along the polymer chain. The Markovian character of Brownian motion reflects the fact that the position of each monomer depends only on the position of its predecessor. The Gaussian chains thus have "zero folding memory". Kuhn's model was the motivation for the present work, where the concept of folding memory will be used in order to distinguish between ensembles of well-structured and (partially) unstructured proteins (IDPs).

\section{PROTEINS AS DISCRETE PATHS}

In the standard discrete path representation of proteins, each residue is represented by its $C_{\alpha}$-atom. In the following, we will both this standard representation and an alternative one, in which secondary structure elements (SSEs) are essentially filtered out. SSEs are characterised by a regular winding of the protein main chain with a typical period between 2 and 4 monomers (residues) and thus lead to a priori "trivial" folding memory effects on that scale. Our straightened path representation replaces SSEs by the axis around which the main chain is wound. This axis can be obtained using the ScrewFrame algorithm. 13 The global fold of a protein is 

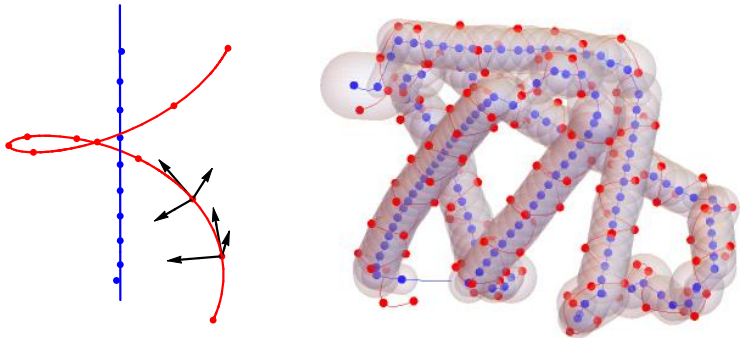

FIG. 1: Left: Exact helicoidal trace of $C_{\alpha}$-atoms (red points) and corresponding screw motion centres (blue points). The figure also shows two consecutive Frenet frames (black arrows), which are attached to $C_{\alpha}$-atoms 2 and 3 , respectively. Right: $C_{\alpha}$-trace and corresponding screw motion centres for myoglobin (PDB structure code 1AB6). The local radius of the gray tube is defined by the radius of the corresponding screw motion.

described as a succession of screw motions aligning successive discrete Frenet frames along the $C_{\alpha}$-trace. The centres for the constructed screw motions then define a "polymer chain" along the protein axis. In contrast to the $C_{\alpha^{-}}$-trace, where the distances between adjacent $C_{\alpha^{-}}$ atoms are nearly constant, $\Delta \approx 0.38 \mathrm{~nm}$, the distances between adjacent screw motion centres vary and are considerably shorter. The protein axis polymer chain may be associated with a Rouse chain, where the monomers are connected by springs.14

The left part of Fig. 1 1illustrates the construction of the screw motion centres (blue points) from a $C_{\alpha}$-trace (red points) which has the form of an ideal helix, such that the corresponding screw motion centres lie on a straight axis (except for the first and the last one). The two Frenet frames shown in black define the screw motion from "monomer" 2 to 3 . For $N C_{\alpha}$-atoms there are $N-1$ screw motion centres. The right part of the figure shows the corresponding analysis for myoglobin (PDB structure code $1 \mathrm{AB} 6$ ). It is important to note that ScrewFrame is applicable to any $C_{\alpha}$-trace, i.e. also to $\beta$-strands, which are "flat helices", and for unstructured parts of a protein. Secondary structure elements are characterised by recurrent screw motion parameters and in particular by a straight axis joining the screw motion centres. The ScrewFrame algorithm leads effectively to a tube model for proteins (indicated in transparent grey), where the local tube axis is defined by the succession of screw motions centres and the local radius by the radius of the respective screw motion. The tube can be considered as excluded volume of the protein main chain. In polymer physics, tube models are used to explain the slow dynamics of reptation, $\frac{15116}{16}$ where the tube represents the space accessible to a single polymer inside the polymer matrix forming its environment, but the reptation model is obviously not a valid picture for the dynamics of protein main chains.

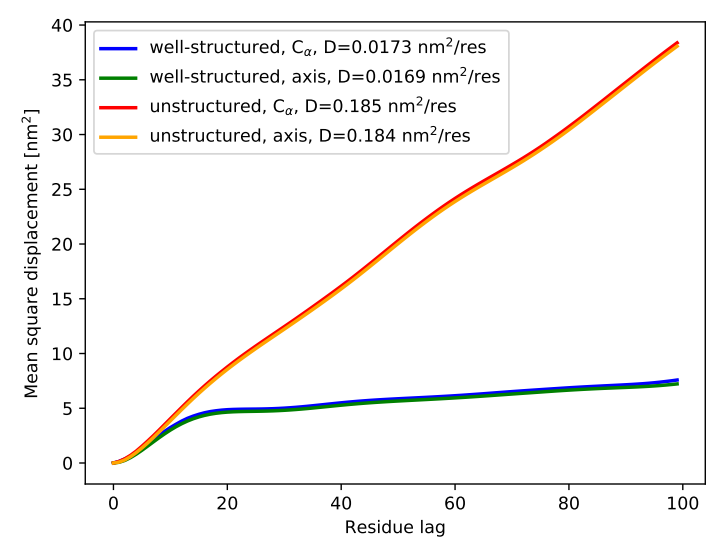

FIG. 2: Mean square displacement as a function of residue lag for well-structured and unstructured proteins. In both cases the MSDs are shown for the $C_{\alpha}$-traces and for the protein axes.

\section{DIFFUSIVITY OF PROTEIN PATHS}

\section{A. Mean square displacements}

Starting from the analogy between polymer models and discrete stochastic paths, we consider first the ensemble-averaged mean square displacement (MSD)

$$
W(n)=\left\langle\frac{1}{N_{x}-n} \sum_{k=0}^{N_{x}-1-n}(\mathbf{x}(k+n)-\mathbf{x}(k))^{2}\right\rangle,
$$

where $N_{x}$ is the number of steps in the discrete path, $\mathbf{x}(k)\left(k=0, \ldots, N_{x}-1\right)$, and $n=0, \ldots, P \ll N_{x}$ to obtain good statistics. For our calculations we used $P=100$. The brackets in (1) denote an average over the protein structures in the given ensemble, where each protein structure counts equally. This weighting scheme, which corresponds to unconstrained maximum entropy weighting $\frac{17}{}$, is very different from thermal averaging of configurations in statistical mechanics, where each configuration is weighted with a Boltzmann factor. Eq. (1) is constructed in complete analogy with time-dependent MSDs, as they are for example calculated from single particle tracking in biological systems or from molecular dynamics simulations. MSDs of discretely sampled trajectories are traced as a function of the time lag $n \equiv n \Delta t$, where $\Delta t$ is the sampling step, whereas the MSDs presented in this paper are traced as function of the dimensionsless "residue lag". In this context, it may appear more appropriate to speak of "mean square distances" instead of "mean square displacements", because we are not considering moving particles. However, we keep the first term in order to maintain the analogy with trajectory analyses. 

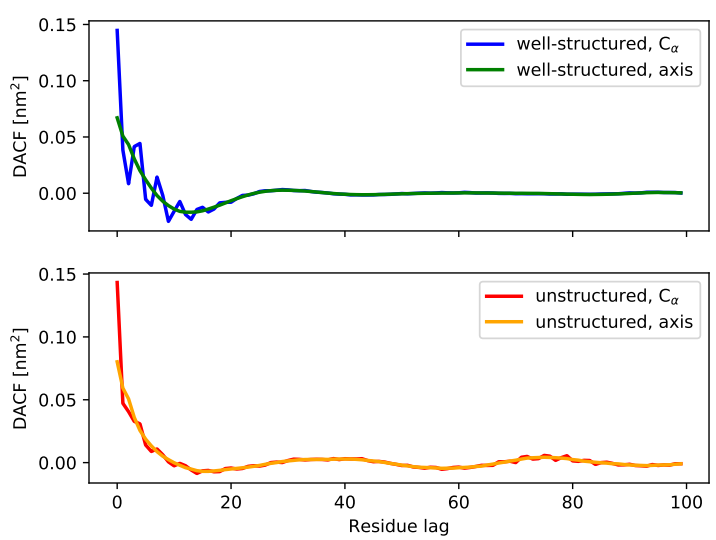

FIG. 3: Upper panel: DACFs for well structured proteins comparing the $C_{\alpha}$-traces and protein axes. Lower panel:The same for unstructured proteins.

Fig. 2 shows the MSD as a function of residue lag for well-structured and unstructured proteins. In the first case we used protein structures from the ASTRAL data base $e^{18 \mid 19}$ and in the second from the pE-DB data base.10 The diffusion coefficients indicated in the plot have been obtained by fitting a linear expression of the form

$$
W(n)=2 D n+a
$$

to the MSD data for $n \geq 20$. This offset appears clearly in the data for well-structured proteins and corresponds roughly to the maximum length of protein secondary structure elements. Practically no differences can be found between the MSDs for the $C_{\alpha}$-trace and the protein axis, but the diffusion coefficient for unstructured proteins is about ten times larger than for well-structured proteins. We find $D \approx 0.18 \mathrm{~nm}^{2} /$ res. in the first case and $D \approx 0.017 \mathrm{~nm}^{2} /$ res. in the second. Using the the polymer-trajectory analogy, the asymptotic linear form of $W(n)$ for both well-structured and unstructured proteins corresponds to "normal diffusion". From this point of view they behave like Gaussian chains or, equivalently, like trajectories of Brownian particles. As we will show in the following, the local behaviour is, however, very different.

\section{B. Displacement autocorrelation functions}

In order to investigate the local properties of our two polymer models for well-structured and unstructured proteins, we make use of a well-known relation between the time-dependent MSD for a diffusing classical particle and its velocity autocorrelation function (VACF), $c_{v v}(\tau)=\langle\mathbf{v}(0) \cdot \mathbf{v}(\tau)\rangle$. Assuming stationarity of the
VACF one derives 20

$$
W(t)=2 \int_{0}^{t} d \tau(t-\tau) c_{v v}(\tau)
$$

were $\langle\ldots\rangle$ denotes a classical ensemble average over the phase space of the diffusing particle. The VACF itself fulfils an equation of motion of the form 21

$$
\dot{c}_{v v}(t)+\int_{0}^{t} d \tau \kappa_{v}(t-\tau) c_{v v}(\tau)=0,
$$

where the memory kernel $\kappa(t) v$ can be formally expressed by the microscopic forces acting on the diffusing particle and between the solvent particles. In the following, only use the general form of the equation of motion (4) is of importance. At the velocity level, the motion of a Brownian particle is described by the Langevin equation, $\dot{\mathbf{v}}(t)+\gamma \mathbf{v}(t)=\mathbf{f}_{s}(t)$, where $\mathbf{f}_{s}(t)$ is white noise, $\gamma>0$ a friction constant. The memory kernel has the form $\kappa_{v}(t)=\gamma \delta(t)$, where $\delta(t)$ is the Dirac delta function. Brownian motion is thus "memory-less" and the VACF has the form $c_{v v}(t)=\left\langle|\mathbf{v}|^{2}\right\rangle \exp (-\gamma t)$. We will now investigate which kind of VACF and corresponding memory function will emerge from the polymer paths representing well-structured and unstructured proteins. The VACF becomes here in fact a discrete displacement autocorrelation function (DACF),

$$
c_{d d}(n)=\left\langle\frac{1}{N_{d}-n} \sum_{k=0}^{N_{d}-1-n} \mathbf{d}(k+n) \cdot \mathbf{d}(k)\right\rangle,
$$

where $\mathbf{d}(k)=\mathbf{x}(k+1)-\mathbf{x}(k)\left(k=0, \ldots, N_{d}-1\right)$ and $N_{d}=$ $N_{x}-1$. Using that the convolution is commutative, the memory function equation (4) is replaced by the discrete version

$$
\Delta c_{d d}(n)+\sum_{k=0}^{P} w(k) c_{d d}(n-k) \kappa_{d}(k)=0,
$$

for $n=0, \ldots, P \ll N_{d}$. Here $w(k)$ are integration weights according to the second order (trapezoidal) rule for numerical integration, $w(0)=w(P)=1 / 2$ and $w(k)=1$ for $k=2, P-1$, and $\Delta$ denotes a numerical derivative of second order. Eq. (6) represents a triangular linear system of equations for $\kappa_{d}(k)(k=0, \ldots, P)$, which can be solved recursively. The second order approximation for numerical integration and differentiation ensures that, to a good approximation, $\kappa_{d}(n) \propto \lambda \delta_{0 n}$, if $c_{d d}(n)=c_{d d}(0) \exp (-\lambda n)$ and $N_{d} \gtrsim 100$.

Figure 3 shows the DACFs for well-structured (upper panel) and unstructured proteins (lower panel). In contrast to the MSDs, there is a clear difference between the DACFs corresponding, respectively, to the $C_{\alpha}$-trace and the protein axis. The DACFs for the protein axis do not display the fast initial oscillations which are seen in the DACFs for the $C_{\alpha}$-trace and which are particularly pronounced for well-structured proteins. These oscillations 

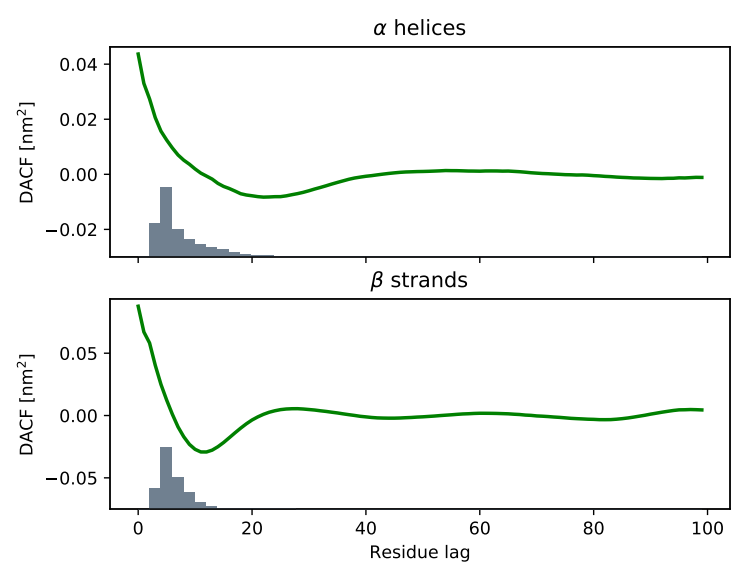

FIG. 4: Upper panel: DACFs for the protein axis of well structured proteins containing essentially $\alpha$-helices and histogram for the lengths of the latter. Lower panel: The same for $\beta$-strands.

can be attributed to the presence of secondary structure elements, in which the direction of the displacements changes periodically with residue lags of approximately 2 ( $\beta$-strands) to 4 ( $\alpha$-helices). The fact that the oscillations are less pronounced for unstructured proteins than for well-structured ones is simply due to the fact that unstructured proteins contain fewer secondary structure elements.

The DACF for the protein axis of well-structured proteins has a striking similarity with the VACF of simple liquids. A surprising result of Rahman's historic simulation of liquid $\operatorname{argon}{ }^{22}$ was that the VACF for such a system does not decay exponentially, as for the Langevin model, but displays damped oscillations which are ascribed to rattling motions of the diffusing molecules in the cage of nearest neighbours. The lag time corresponding to the first minimum corresponds here to the typical time for a reversal of its velocity. In analogy, the DACF for the protein axis of well-structured proteins displays a pronounced minimum for residue lags of about $n=18$, which means that the displacement vector $\mathbf{d}$ tends to invert its direction after 18 consecutive steps. The ScrewFrame protein axis may here be considered as an analogue of a simulated (discrete) Molecular Dynamics trajectory. Knowing that typical secondary structure elements have about a length of 18 , such a behavior could be explained by the typical "helixloop-helix" successions in well-structured proteins such as myoglobin. The term "helix" must here be understood in the sense of the ScrewFrame algorithm, i.e. as a regular secondary structure element, a category that includes $\alpha$-helices and $\beta$-strands. To investigate this point in more detail, we have computed the DACFs for the protein axis of well-structured proteins separately for sub-

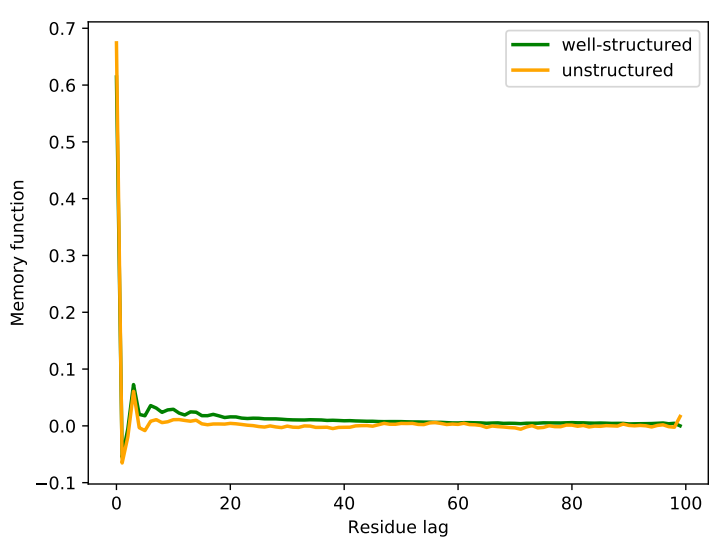

FIG. 5: Memory kernel of the protein axis DACF for wellstructured proteins and unstructured proteins.

ensembles of protein structures containing, respectively, essentially $\alpha$-helices and $\beta$-strands and recording in both cases histograms for the lengths of these secondary structure elements. Fig. 4 shows clearly that the first minima of the axis DACFs are correlated with the maximum lengths of the secondary structure elements, which confirms the hypothesis that the first minimum of the DACF reflects effectively the recurrent "helix-loop-helix" motif in globular well-structured proteins. Since this motif is less present in IDPs, the minimum of the corresponding protein axis DACF is less pronounced.

The frequency of the "helix-loop-helix" motif should also be reflected in the memory kernel of the DACF. Fig. 5 shows the memory kernels for protein axis DACFs of well-structured proteins and unstructured proteins. Although the difference is small, it is systematic: The memory function corresponding to the DACF of wellstructured is systematically larger than its counterpart for unstructured proteins, indicating stronger "folding memory". The slight oscillations in the latter case should not be overinterpreted since they might be artefacts due to insufficient statistics.

\section{CONCLUSIONS}

Our study shows that suitably defined polymer models for proteins enable a meaningful statistical analysis of their folding properties on the basis of "polymer paths". Each path is here a succession of points that represent the residues, and two types of paths are considered: 1) the $C_{\alpha}$-representation, where each residue is represented by its $C_{\alpha}$-atom, and 2) the ScrewFrame representation, where each residue is represented by the projection of the $C_{\alpha}$ position onto an appropriately constructed protein main axis. The resulting paths are analyzed within 
a theoretical framework that is inspired by the theory of the generalized Langevin equation. We show in particular that the memory functions associated with the displacement autocorrelation function along the protein chain display much stronger "folding memory" for wellstructured proteins than for IDPs. Although the statistical basis for unstructured proteins is still fairly small, the theoretical framework allows for discriminating between ensembles of well-structured and unstructured proteins. The next step will be to develop suitable simple memory function models which explain the the data at least semiquantitatively and which have a physical interpretation.

\section{Supplementary Material}

See supplementary material for the complete source code of our analysis software with the input and output datasets.
${ }^{1}$ R. H. Austin, K. W. Beeson, L. Eisenstein, H. Frauenfelder, and I. C. Gunsalus, Biochemistry 14, 5355 (1975).

${ }^{2}$ N. Alberding, R. H. Austin, S. S. Chan, L. Eisenstein, H. Frauenfelder, I. C. Gunsalus, and T. M. Nordlund, The Journal of Chemical Physics 65, 4701 (1976), ISSN 00219606, 1089-7690.

${ }^{3}$ H. Hartmann, F. Parak, W. Steigemann, G. Petsko, D. Ponzi, and H. Frauenfelder, P Natl Acad Sci Usa 79, 4967 (1982).

${ }^{4}$ H. Frauenfelder, S. G. Sligar, and P. G. Wolynes, Science 254, 1598 (1991).

${ }^{5}$ H. J. Dyson and P. E. Wright, Nature Reviews Molecular Cell Biology 6, 197 (2005), ISSN 1471-0072, 1471-0080.

${ }^{6}$ R. B. Best, Current Opinion in Structural Biology 42, 147 (2017), ISSN 0959440X.

7 A. Soranno, A. Holla, F. Dingfelder, D. Nettels, D. E. Makarov, and B. Schuler, Proceedings of the National Academy of Sciences 114, E1833 (2017), ISSN 0027-8424, 1091-6490.

8 P. Kulkarni and V. N. Uversky, PROTEOMICS 18, 1800061 (2018), ISSN 16159853.

${ }^{9}$ P. W. Rose, A. Prli, C. Bi, W. F. Bluhm, C. H. Christie, S. Dutta, R. K. Green, D. S. Goodsell, J. D. Westbrook, J. Woo, et al., Nucleic Acids Research (2014).

10 M. Varadi, S. Kosol, P. Lebrun, E. Valentini, M. Blackledge, A. K. Dunker, I. C. Felli, J. D. Forman-Kay, R. W. Kriwacki, R. Pierattelli, et al., Nucleic Acids Research 42,
D326 (2014), ISSN 0305-1048, 1362-4962.

11 M. Necci, D. Piovesan, Z. Dosztányi, P. Tompa, and S. C. E. Tosatto, Bioinformatics 34, 445 (2018), ISSN 1367-4803, 1460-2059.

12 W. Kuhn, Kolloid-Zeitschrift 68, 2 (1934), ISSN 0303402X, 1435-1536.

13 G. R. Kneller and K. Hinsen, Acta Crystallogr D 71 (2015).

14 P. E. Rouse, The Journal of Chemical Physics 21, 1272 (1953), ISSN 0021-9606, 1089-7690.

15 P. G. de Gennes, The Journal of Chemical Physics 55, 572 (1971), ISSN 0021-9606, 1089-7690.

16 T. C. B. McLeish, Advances in Physics 51, 1379 (2002), ISSN 0001-8732, 1460-6976.

17 A. Papoulis, Probablity, Random Variables, and Stochastic Processes (McGraw Hill, New York, 1991), 3rd ed.

18 J.-M. Chandonia, G. Hon, N. S. Walker, L. Lo Conte, P. Koehl, M. Levitt, and S. E. Brenner, Nucleic Acids Research 32, D189 (2004).

19 N. K. Fox, S. E. Brenner, and J. M. Chandonia, Nucleic Acids Research 42, D304 (2013).

20 J. Boon and S. Yip, Molecular Hydrodynamics (McGraw Hill, New York, 1980).

21 R. Zwanzig, Nonequilibrium statistical mechanics (Oxford University Press, 2001).

22 A. Rahman, Physical Review 136, A405 (1964), ISSN 0031-899X. 